\title{
Convergence Analysis of Finite Element Approach to Classical Approach for Analysis of Plates in Bending
}

\author{
Ferdinand Niyonyungu ${ }^{1 *}$, Jules Karangwa ${ }^{2}$ \\ 1 School of Civil Engineering, Lanzhou Jiaotong University, Lanzhou Gansu China \\ 2 Department of construction and structural engineering, University of Dar Es salaam, Tanzania \\ * Corresponding author's e-mail: niol20@yahoo.fr
}

\begin{abstract}
Classical approach is a popular method used in the analysis of structures including bending plates, but these plates can have highly irregular geometry and contain holes or may be subjected to loading irregularity. Hence, the analysis is further complicated and the classical approaches are not valid. Thus, the Finite Element Method is used to control the accuracy and it is needed for more difficult problems. In the present study Fourier series theory as classical approach and finite element method of analysis were discussed and the numerical examples of a simply supported and fixed supported square steel plate were used to compare them. The results obtained with general public software LISA that uses FEM was plotted according to the element types, both quadrangular and triangular. Their convergence was verified with the values obtained from classical approach to validate the results of FEM from LISA. The results showed the conformity with the existing theories that the greater discretization the more the reality is approached. The convergence error of 5\% was taken as the maximum for the element types and meshes to be used for highly sophisticated plate systems. The ratios of element size to the size of the whole square plate to be used for general cases of square plate dimensions were established.
\end{abstract}

Keywords: classical methods, finite elements, bending plates, bending moment, RISA

\section{INTRODUCTION}

There are two important properties that define a plate structure; first by considering the geometrical configuration, a plate structural is a three dimensional solid with a very small thickness compared to other two planar dimensions. Secondly, the forces applied on a plate are perpendicular to the plane of the plate. Therefore, a plate resists the applied load by means of bending in two directions and twisting moment. By considering the structural plate characteristics, a plate theory was used to transform a 3D problem into 2D. The plate theory has the specific aim of calculating the deformation and stresses in a plate when subjected to loads [1].

Due to the practical importance of plate structures, engineers have long been faced with the task of analyzing plates of various geometry and loading. Unfortunately, the governing differential equations are solvable only for simple geometry and boundary conditions [2]. During the last ten years, much progress has been made in the development of structural methods of analysis based on matrix algebra and a discretization of the structure into an assembly of discrete structural elements. In these methods, a displacement or a stress distribution is assumed within the element and a complete solution is then obtained by combining this approximate displacement or stress distributions in a manner which satisfies the force-equilibrium and displacement-compatibility requirements at all interfaces of the elements [2]. The methods based on such approaches have been proven to be suitable for the analysis of complex structures. This gave rise to the development of the finite element methods. 
These methods have been proven successful and used in analysis of many complex civil engineering planar structures like thick plates and slabs and other non-planar forms [3]. However, through finite element method, internal forces and moments are determined and afterwards the design is done by referring to design codes.

Finite element analysis has been used by engineers since 1960s [4] and the theory behind such method has been well-explored by numerous researchers. As the time goes on, the human has started to build complex structures geometrically and in load distribution. The analysis of such structures by using classical methods had become very difficult and time consuming when considered time constraints and FE has seemed to be the answer for designing complex structures within short time [5].

Despite numerous studies associated with finite element based flat plate analysis, the finite element method is not without limitations. While finite element is very effective in handling complexities that restrict simplified design methods, finite elements exhibit several important practical constraints. First of all, there is a difficulty in the interpretation of the results of finite element analysis are and unsuitability in their direct use. In order to interpret the results, understanding of several sign conventions and coordinate system is necessary.

It is very common for a structural model to contain millions finite elements and hundred or more loading cases.

The geometrical irregularity and complexity of boundary conditions of plates makes the analysis, results interpretation and reduction of that complexity to a simple design difficult for an engineer. This is due to the fact that in finite element analysis, each element contains more nodes and, in their turn, those nodes contain more degree of freedom which results in voluminous results in this analysis. Today's practice is based on the determination of design forces along a cross section on an element by element basis, usually node by node per element. All loading conditions must be checked to determine the maximum effect and reduced to a design envelope. There is a high possibility of errors on the part of the engineer when this method is used.

The limitations associated by the use of finite element method was described by Hrabok and Hrudey and Alison E. Hatheway [22. 23] where they mentioned that the choice of elements for analysis including various shapes, configurations of nodal and nodal degree of freedom were the most important. The lack of sufficient training in this method complicates this matter even further.

The modeling and analysis of plates in bending using the classical approach is certainly true for the plates loaded uniformly and with regular geometry. If the plate has highly irregular geometry and contains holes or is subjected to concentrated or otherwise irregular loadings, the analysis is further complicated and the classical approaches are not valid. Thus, the Finite Element Method were proven to be the most powerful numerical techniques ever devised for solving differential (and integral) equations of initial and boundary-value problems geometrically complicated regions" [6].

The objective of this research was to emphasise the use of finite element methods of analysis for the plate structures in bending by comparing it with classical analysis approaches. Specifically, this paper assessed the percentage of convergence errors of finite element method to classical method of analysis for flat plates in bending, and the restrictions of classical analysis approach was highlighted to recommend the use of finite element method in the design of flat plate in bending.

The scope of this study was limited to the comparison of two methods of analysis; classical method and Finite Element method for plates in bending. Two square plates were used as example one simply supported from all sides and the other clamped from all sides with uniform loading, the classical approach was performed first and the modeling with LISA software that uses Finite Element analysis method was also performed. The convergence of the results from LISA was checked compared to the classical approach results to draw a conclusion and Recommendations.

\section{TRADITIONAL ANALYSIS METHODS FOR FLAT PLATES}

\section{Plate theory}

By this theory, three assumptions are used to reduce the equations of 3D theory of elasticity to $2 \mathrm{D}$. First, the line normal to the neutral axis before bending remains straight after bending, second, the normal stress in thickness direction is neglected, i.e. this assumption converts the $3 \mathrm{D}$ problem into a $2 \mathrm{D}$ problem. $\sigma z=0$ and third, 
the transverse shearing strains are assumed to be zero. i.e., shear strains $\gamma \mathrm{xz}$ and $\gamma \mathrm{yz}$ will be zero. Thus, the thickness of the plate remains constant during bending.

\section{Moment equations of the bending plate}

By considering a plate element of $d x \times d y$ and with thickness $t$, the plate is subjected to external uniformly distributed load $P$. For a thin plate, body force of the plate can be converted to an equivalent load and therefore, consideration of separate body force is not necessary.

From the relation above, it can be observed that stresses vary linearly along thickness of the plate (Fig. 2). Hence the moments (Fig. 3) on the cross section can be calculated by integration.

$$
\begin{aligned}
& M=\left[\begin{array}{c}
M x \\
M y \\
M x y
\end{array}\right]=\int_{\frac{t}{2}}^{\frac{t}{2}} \sigma z d t= \\
& =\left(\int_{\frac{t}{2}}^{\frac{t}{2}} z^{2} d t\right)[D] \Delta^{2} W=-\frac{t^{3}}{12}[D] \Delta W \\
& M_{x}=\frac{E t^{3}}{12\left(1-v^{2}\right)}\left(\frac{\partial^{2} w}{\partial x^{2}}+v \frac{\partial^{2} w}{\partial y^{2}}\right)= \\
& =D_{p}\left(\chi_{x}+v \chi_{y}\right) \\
& M_{y}=\frac{E t^{3}}{12\left(1-v^{2}\right)}\left(\frac{\partial^{2} w}{\partial y^{2}}+v \frac{\partial^{2} w}{\partial x^{2}}\right)= \\
& =D_{p}\left(\chi_{y}+v \chi_{x}\right) \\
& M_{x y}=M_{y x} \frac{E t^{3}}{12\left(1-v^{2}\right)}\left(\frac{\partial^{2} w}{\partial x y}\right)= \\
& =D_{p}\left(\frac{1-v}{2}\right) \chi_{x y}
\end{aligned}
$$

where $D_{p}$ is flexural rigidity of the plate and is given by

$$
D p=\frac{E t^{3}}{12\left(1-v^{2}\right)}
$$

\section{SOLUTION BY FOURIER SERIES OF PLATES}

\section{Fourier series theory}

The Fourier series help determining the value of transversal deflection at the middle plane of the plate $(\mathrm{w})$ which once determined the bending moment Mx, My and twisting moment Mxy can be calculated using Equation (2.10)

In general each edge may be simply supported (S), clamped (C) or free (F), so there are 21 different possible combinations of boundary conditions, which are listed in Table 1, where the Reddy notation of the boundary condition is adopted and the consecutive pair of letters indicates a boundary condition on opposite edges ([7], p. 266). Note that the cases below the diagonal in Table 1 are obtained by rotating a plate by $90^{\circ}$. For solving the problem, different authors introduced the simplistic method, which reduces a two-dimensional plate problem to an Eigenvalue problem. According to the historical notes of Love [8], Timoshenko et al. [9] and Melelshko [10], the first SSSS plate problem was solved by Navier (1823) by using a double trigonometric series. Later, Lévy (1899) provided a single trigonometric series solution of a plate which has two opposite edges simply supported, The FFFF plate was solved by Galerkin (1915) as a limit case of a plate with elasticity supported edges [9]. A recent solution using the simplistic method was given by Lim et al. [11] using the Fourier method [12] where a set of references is provided for the values of deflection and moments in selected referenced points of the plate.

\section{General considerations}

Consider a homogeneous isotropic elastic rectangular thin plate of sides $a^{\prime}=2 \mathrm{a}$ and

Table 1. Possible combination of boundary conditions. Bold indicates the cases discussed by Timoshenko et al. [9]

\begin{tabular}{|l|l|l|l|l|l|l|}
\hline SS & SSSS & SSSC & SSSF & SSCC & SSCF & SSFF \\
\hline SC & & SCSC & SCSF & SCCC & SCCF & SCFF \\
\hline SF & & & SFSF & SFCC & SFCF & SFFF \\
\hline CC & & & & CCCC & CCCF & CCFF \\
\hline CF & & & & & CFCF & CFFF \\
\hline FF & & & & & & FFFF \\
\hline
\end{tabular}


$b^{\prime}=2 \mathrm{~b}$ subject to a uniformly distributed load $q$. The Cartesian coordinate system Oxy is originated at the center of the plate and the plate is orientated in the way that it occupies the region $-\mathrm{a} \leq \mathrm{x} \leq \mathrm{a},-\mathrm{b} \leq \mathrm{y} \leq \mathrm{b}$. The governing equation of the plate $([9]$, p. 82$)$ is

$$
\Delta W=q / D
$$

The equation (2.12) should be solved in such a way that the boundary conditions at the edge of the plate are satisfied, for a symmetrical boundary condition the solution of governing equation (2.12) should be symmetrical in $x$ and $y$. The symmetrical solution of equation (2.12) obtained by the Fourier method of separation of variables [13] may be written in the form.

$$
\begin{aligned}
& w=w_{o}+\frac{q}{D} \sum_{n=0}^{\infty}(-1)^{n}\left(A_{n} \frac{\cosh \alpha_{n} y}{\cosh \alpha_{n} b}+B_{n} \frac{y \sinh \alpha_{n} y}{b \cosh \alpha_{n} b}\right) \cosh \alpha_{n} \\
& -\frac{q}{D} \sum_{n=0}^{\infty}(-1)^{n}\left(C_{n} \frac{\cosh \beta_{n} x}{\cosh \beta_{n} a}+D_{n} \frac{x}{a} \frac{\sinh \beta_{n} y}{\cosh \beta_{n} a}\right) \cosh \beta_{n} y
\end{aligned}
$$

where $w_{0}$ is a particular solution satisfying

$$
\begin{aligned}
\Delta W & =q / D \text { and where } \\
\alpha_{n} & =\left(\frac{2 n+1}{2 a}\right) \pi \\
\beta_{n} & =\left(\frac{2 n+1}{2 b}\right) \pi n=1,2,3, \ldots
\end{aligned}
$$

The particular solution $w_{0}$ is taken in the form of a symmetrical polynomial of the fourth order in $x$ and $y$

$$
w_{0}=c_{o}+c_{1} x^{2}+c_{2} y^{2}+c_{3} x^{4}+c_{4} x^{2} y^{2}+c_{5} y^{4}
$$

This solution must satisfy the plate equation, so

$$
3 \mathrm{c}_{3}+\mathrm{c}_{4}+3 \mathrm{c}_{5}=\frac{q}{8 D}
$$

The simplified equations were established according to the ratio between two sides of a plate due to their boundary conditions.

Simply supported square plate

Case 1: Displacement

$$
w=\frac{\alpha s \times q \times a 4}{D}
$$

Where $\alpha s$ represent the numerical value coefficient which include simply boundary condition problem $q$ represent uniformly distributed load $D$ represent the flexural rigidity of the plate,

$$
\begin{aligned}
& D=\frac{E t^{3}}{12\left(1-v^{2}\right)} \\
& \mathrm{a} / \mathrm{b}=1, \alpha s=0.00406235
\end{aligned}
$$

Case 2: Bending moment

$$
M x=M y=\beta s \times q \times a^{2}
$$

Where $\beta$ s represent the numerical value coefficient which include simple boundary condition problem $q$ represent uniformly distributed load $\mathrm{a} / \mathrm{b}=1, \beta s=0.0478864$

Fixed supported square plate

Case 1: Displacement

The case of fixed supported square plate is considered and the only change is the coefficient $(\alpha f)$ that includes the fixed boundary condition.

$$
w=\frac{\alpha f \times q \times a 4}{D}
$$

Where $\alpha f$ represent the numerical value coefficient which include fixed boundary condition problem

$\mathrm{a} / \mathrm{b}=1, \alpha f=0.00126532$

Case 2: Bending moment

The only change is numerical value coefficient $(\beta f)$ which include fixed boundary condition problem

$$
\begin{aligned}
& \mathrm{a} / \mathrm{b}=1, \beta f=0.0229051 \\
& M x=M y=\beta f \times q \times a^{2}
\end{aligned}
$$

\section{FINITE ELEMENT METHOD}

The finite element method is an approximation in which a continuum is replaced by a number of discreet elements [14]. Each component representing the system as a whole is known as a finite element. Parameters and analytical functions describe the behavior of each element and then are used to generate a set of algebraic equations describing the displacements at each node, which can then be solved. The elements have a finite size and therefore the solution to these equations is approximate; the smaller the element, the closer the approximation is to the true solution [15].

The finite element method can be considered a convenient instrument for the resolution of the problems which are governed by a system 
of partial differential equations. In the problems of linear elasticity of the mechanics of solids and structures, the most common formulation employed consists in expressing the equilibrium differential equation in terms of displacement as the only independent field variable. The corresponding displacement formulation in the finite element method is based on the variation equation given by the minimum Total Potential Energy (TPE).

A brief summary of the linear elastic governing equations where the Finite Element equations are derived are described below by considering a body that occupies the region $\bar{B}$ in Cartesian coordinate $\mathrm{x}, \mathrm{y}, \mathrm{z} . \bar{B}$ is formed of $\mathrm{BU} B U \partial B$ i.e. set of points within the domain of $\mathrm{B}$ and boundary of $\mathrm{B}, \partial B$.

$$
\begin{array}{ll}
{\left[\begin{array}{l}
u x \\
u y
\end{array}\right]\left[\begin{array}{l}
\bar{u} x \\
\bar{u} y
\end{array}\right]} & \text { Compatibility } \\
\underline{u}=\bar{u} \operatorname{In} \partial B & \\
{\left[\begin{array}{c}
\varepsilon x \\
\varepsilon y \\
\gamma x y
\end{array}\right]=-z\left[\begin{array}{l}
\partial^{\wedge} 2 / \partial^{\wedge} 2 x \\
\partial^{\wedge} 2 / \partial^{\wedge} 2 y \\
\partial^{\wedge} 2 / \partial x \partial y
\end{array}\right] w} & \text { Hooke's law } \\
\underline{\varepsilon}=\underline{D} \cdot \underline{u} \operatorname{In} \mathrm{B} & \\
{\left[\begin{array}{ccc}
\partial / \partial x & \partial / \partial y & 0 \\
0 & \partial / \partial x & \partial / \partial y
\end{array}\right]\left[\begin{array}{c}
\sigma x \\
\lambda x y \\
\sigma y
\end{array}\right]=\left[\begin{array}{l}
p x \\
p y
\end{array}\right]} & \text { Equilibrium } \\
\underline{D} \cdot \underline{\sigma}=p_{x y} \text { In B } &
\end{array}
$$

\section{Total potential energy}

These variational methods form the basis for the derivation which includes all the equations for the linear theory of elasticity. The principle of minimum total potential energy is a result of the work of a Chinese scholar Hu and a Japanese scholar, K.Washizu [17] known as Hu-Washizu varitional theorem which is expressed as,

$$
\begin{gathered}
\prod_{B}(u, \sigma, \varepsilon)=\frac{1}{2} \int_{B} \underline{\varepsilon}^{T} \underline{H \varepsilon} d V-\int_{B} \underline{\varepsilon}^{T} \underline{H \varepsilon} d V+ \\
+\int_{B} \underline{\sigma}^{T}(\underline{D u}-\varepsilon) d V-\int_{B} \underline{u} \underline{p} d V-\int_{B} \underline{u}^{T} \underline{t} d- \\
-\int_{\partial B u}(\underline{u}-\underline{\bar{u}})^{T} t d S
\end{gathered}
$$

A variation theorem is stationary when the argument $\underline{u}, \underline{\sigma}, \underline{\varepsilon}$ satisfies the condition where the first variation disappear /vanishes.

From the equation above we can form a basis of various variational formulations such as the
Hellinger-Reissner variational theorem, which is basically a two field formulation in u displacement and $\sigma$ stress as well as the minimum Potential Energy, which is a displacement based variation theorem. The latter will constitute the point departure for the displacement based finite element.

Taking equation (11) assume that the compatibility condition in $\mathrm{B}$ and on $\partial B$ and Hooke's law are satisfied a priori i.e.

$$
\begin{array}{ll}
\underline{\sigma}=H \cdot(\underline{\varepsilon}-\bar{\varepsilon}) & \text { In Bs } \\
\underline{\varepsilon}=\underline{D} \cdot \underline{u} & \text { In B } \\
\underline{u}=\underline{\bar{u}} & \text { On } \partial B
\end{array}
$$

Thus, the function becomes;

$$
\begin{aligned}
\prod(u)= & \frac{1}{2} \int_{B}(\underline{D u})^{T} \underline{H}(\underline{D u}) d V-\int_{B}(\underline{D u})^{T} \underline{H} \bar{\varepsilon} d V- \\
& -\int_{B} \underline{u^{T}} \underline{p} d V-\int_{\partial B t} \underline{u^{T}} \underline{t d} S
\end{aligned}
$$

This constitutes the principal of minimum potential energy and is often used as the basis for developing the displacement finite element method.

\section{Element stiffness equations}

In order to obtain the finite element stiffness equation, the variational of TPE functional is decomposed into contributions from individual elements:

Thus

$$
\begin{gathered}
\prod(u)=\sum_{1}^{m}\left\{\frac{1}{2} \int_{B e}(D u)^{T} H(D u) d V-\right. \\
\left.-\int_{B e}(D u)^{T} H \varepsilon d V-\int_{B e} \underline{u^{T}} \underline{p} d V-\int_{\partial B e t} \underline{u^{T}} \underline{t} d S\right\}
\end{gathered}
$$

Then, the finite element approximation for displacement is given by;

$$
\underline{u}=\sum \underline{N}_{i} \underline{q}_{i}
$$

Where $\underline{N}=\underline{N_{B}}$

$N_{B}$ Is the shape function for plate in bending

$q$ is the free parameters of displacements at the nodes to be determined

From $\underline{u}=\underline{N} \cdot \underline{q}$

Then $\underline{D} \cdot \underline{u}=\underline{D} \cdot \underline{N} \cdot \underline{q}=$ D.N. $q=\underline{B} \cdot q$

Then $\underline{B}=\underline{D} \cdot \underline{N}$ 
Inserting the relation above (3.5) into (3.3) and taking $\Pi$ for an element $\Pi^{e}$ we have;

$\Pi^{e}(u) \cong \frac{1}{2} \int_{B e} q^{T}\left(B^{T} H B\right) q d V-$

$-\int_{B e} q^{T}(B H \bar{\varepsilon}) d V-\int_{B e} q^{T}\left(N^{T} p\right) d V-\int_{\partial B e t} q^{T} \underline{N} \bar{t} d S$

$\Pi^{e}(q)=1 / 2 q^{T} K_{e} q-q^{T} G_{e}-q^{T} F_{e}-q^{T} \bar{F}$

Where $\underline{K}_{e}=\int_{B} \underline{B} \cdot \underline{H} \cdot \underline{B} \cdot d V-$ stiffness matrix of $\underline{G}_{e}=\int_{B} \underline{B} \cdot \underline{H} \cdot \underline{\bar{\varepsilon}} \cdot d V$ - vector of equivalent nodal distortions

$F_{e}=\int_{B} \underline{N}^{T} p \cdot d V$ - vector equivalent nodal loads applied (volume)

$\underline{\bar{F}}_{e}=\int_{B} \underline{N}^{T} \underline{\underline{t}} \cdot d V$ - equivalent loads on nodal boundary

Stationary condition of $\Pi^{e}$ respect to $\mathrm{q}$ for such sub domain is

$$
\begin{aligned}
& \forall q, \partial q, \delta \Pi(q)=\underline{K}_{e} \underline{q}-\underline{G}_{e}-F_{e}-\bar{F}_{e} \\
& =\underline{K}_{e} \underline{q}-f=0
\end{aligned}
$$

Therefore, the consistent element nodal force vector is

$$
f=\underline{K_{e}} \underline{q}
$$

where $f=\underline{G}_{e}+F_{e}+\underline{F}_{e}$

For the stiffness matrix of the element (see eqn. 3.6), we have

$$
\underline{K}_{e}=\int_{B} \underline{B}^{T} \cdot \underline{H} \cdot \underline{B} \cdot d V
$$

But

$$
B=B_{B}
$$

$$
\begin{aligned}
& B_{B}=D_{B} \cdot N_{B} \text { and } \\
& \underline{K}_{e}=\int_{B} B_{B}{ }^{T} \cdot H_{B} \cdot B_{B} d V \\
& \underline{K}_{e}=\int_{B} K_{B} d V
\end{aligned}
$$

where

where $\underline{K}_{B}-$ Stiffness matrix for plate in bending

\section{CASE STUDY}

\section{Characteristics of the model}

A 2D steel square plate of sides $1.0 \mathrm{~m}$ by $1.0 \mathrm{~m}$ with the thickness of $15 \mathrm{~mm}$ was adopted for this study (Table 2).

The plate is assumed to be homogeneous and isotropic; the loading condition is taken as uniformly distributed in the $\mathrm{x}$ and $\mathrm{y}$ directions, orthogonal to the plane of the plate as shown on the Figure 1.

Table 2. Properties of the model

\begin{tabular}{|l|c|}
\hline \multicolumn{1}{|c|}{ Properties } & Value \\
\hline Plate dimension & $1 * 1 \mathrm{~m}$ \\
\hline Thickness $(\mathrm{t})$ & $15 \mathrm{~mm}$ \\
\hline Young's modulus (E): & $205,000 \mathrm{~N} / \mathrm{mm}^{2}$ \\
\hline Poisson's ratio $(\mathrm{v}):$ & 0.3 \\
\hline
\end{tabular}

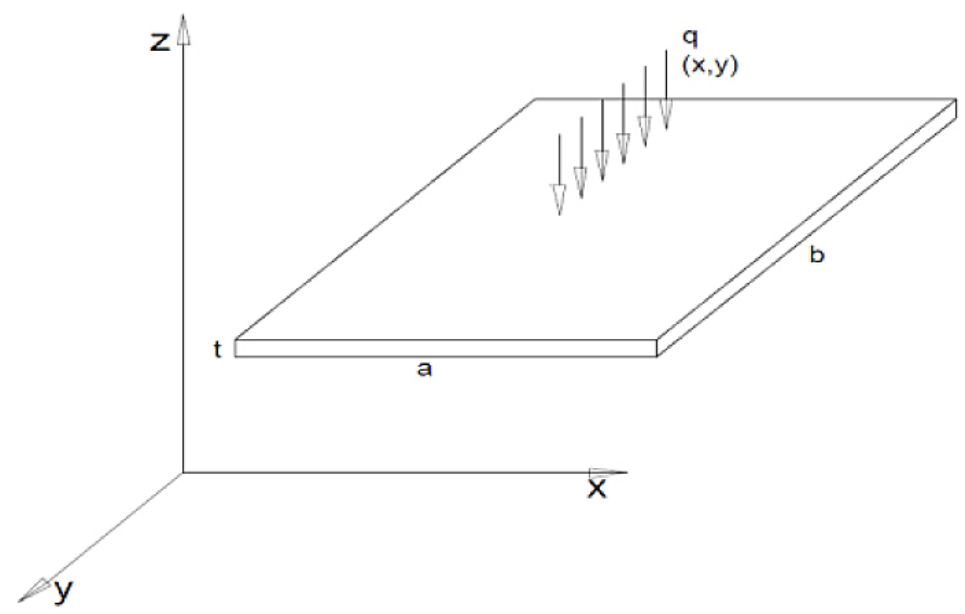

Figure 1. Square isotropic plate with properties and loading 
Two supports conditions were considered, simply supported and fixed. In this project, a 3D static solution was used to run the analysis of the model as it includes the displacement and stresses in the middle plane of the plate.

Quadrangular 2D element with 4, 8 and 9 nodes and triangular 2D element with 6 nodes were also used to mesh the model; the plate was analyzed using different models, each with an increasing number of finite elements. Some of these models are shown in Figures 4 and 5. The four meshes contained; 16 elements $(4 \times 4$ mesh), 64 elements ( $8 \times 8$ mesh), 144 elements $(12 \times 12$ mesh), 256 elements (16x16 mesh), and 400 elements (20 x 20 mesh), respectively. Additionally, a triangular $2 \mathrm{D}$ element with 6 nodes was used with 32 elements ( $4 \times 8$ mesh), 128 elements (16x8 mesh), 288 elements (18x16 mesh) and 512 elements (16x32 mesh).

\section{Result analysis}

A finite element software LISA was used in this paper (Figures 2 and 3).

For each element in the tables below, the value of error expressed in percentage was calculated to show how far the approximated value for each element in each case is close to meet the classical value or exact value. The errors were calculated as follows.
$\operatorname{ERROR}(E)=$

$\left(\frac{\text { EXACT,VALUE }- \text { APPROXIMATE,VALUE }}{\text { EXACT,VALUE }}\right)($<smiles>[GeH2]=[W]</smiles>

The exact value is that from Fourier series method and approximate value is from finite element method (LISA analysis results)

Simply supported square plate

Quadrangular elements

Refer to the equation 2.5, the exact value of center displacement is $W_{\text {exact }}=6.41170 e^{-5}(\mathrm{~m})$

Refer to the equation 2.16, the exact value of bending moment is $M x_{\text {exact }}=M y_{\text {exact }}=47.9 \mathrm{Nm}$.

\section{Triangular elements}

Refer to the equation 2.5, the exact value of center displacement is $W_{\text {exact }}=6.41170 e^{-5}(\mathrm{~m})$

Refer to the equation 2.16, the exact value of bending moment is

$$
M x_{\text {exact }}=M y_{\text {exact }}=47.9 \mathrm{Nm}
$$
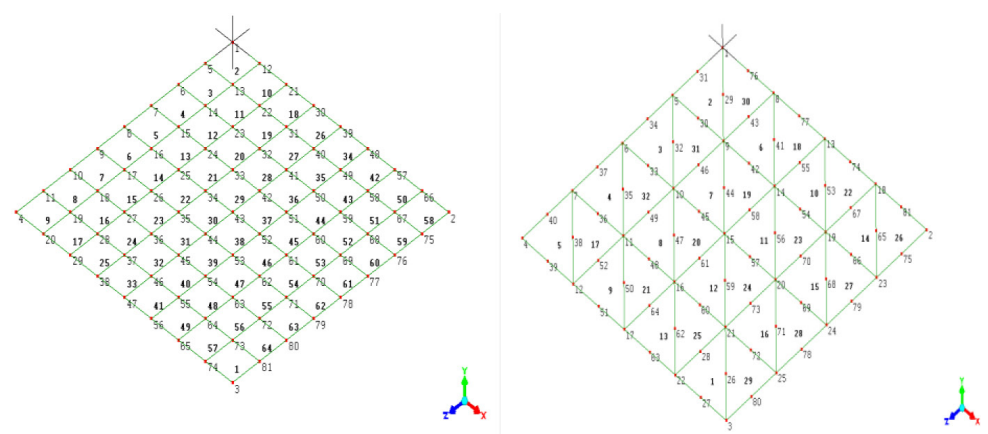

Figure 2. Discretization for both quadrangular and triangular elements
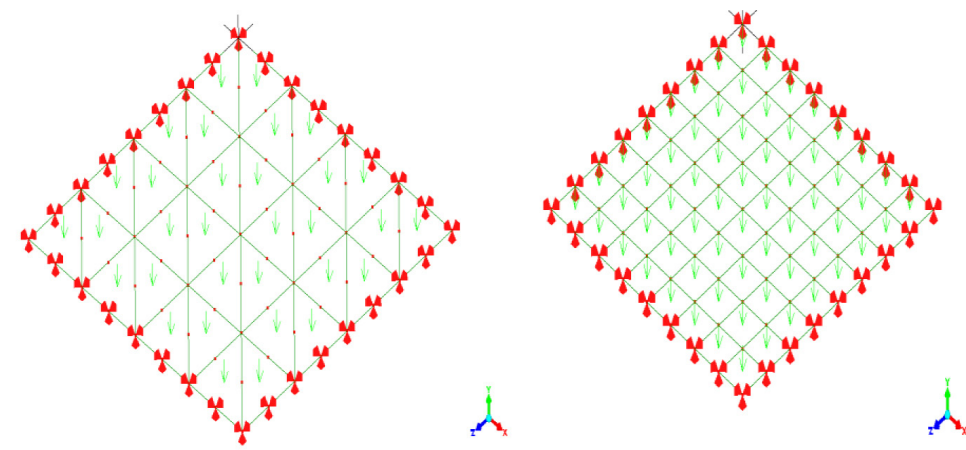

Figure 3. Constraint (simple) and loading for both quadrangular and triangular elements 


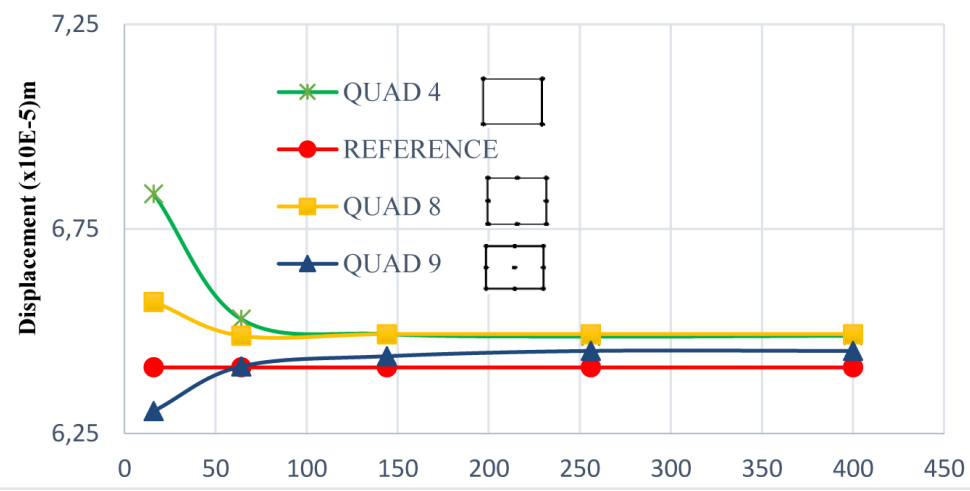

Figure 4. Graph of Displacement vs Number of elements for quadrangular simply supported

\section{Fixed supported square plate}

Quadrangular elements

Refer to the equation 2.17 , the exact value of center displacement is.

$$
W_{\text {exact }}=1.99708 e^{-5}(m)
$$

Refer to the equation 2.8, the exact value of bending moment is $M x_{\text {exact }}=M y_{\text {exact }}=22.9 \mathrm{Nm}$

\section{Triangular elements}

Refer to the equation 2.7, the exact value of center displacement is $W_{\text {exact }}=1.99708 e^{-5}(\mathrm{~m})$.

Refer to the equation 2.8 , the exact value of bending moment is $M x_{\text {exact }}=M y_{\text {exact }}=22.9 \mathrm{Nm}$

\section{CONCLUSION}

Critical evaluation of the results from finite element method is necessary before being relied upon in any application or before being applied, because this method is based on approximation. For both cases, the triangular element exhibits a large error for the first mesh but it ends up with an accurate approximation as those of quadrangular elements, which conform to the theories that as the number of elements increases, the discretized system approaches the reality. In conclusion, the targeted accuracy to recommend elements and mesh size for use was assumed by considering the economical and quality factors. The maximum error of $5 \%$ error was chosen.

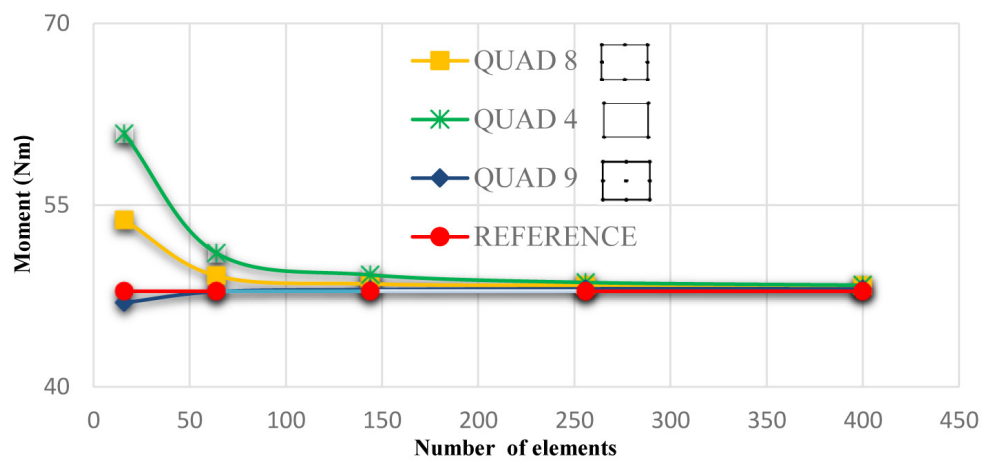

Figure 5. Graph of Bending Moment vs number of elements for quadrangular, simply supported

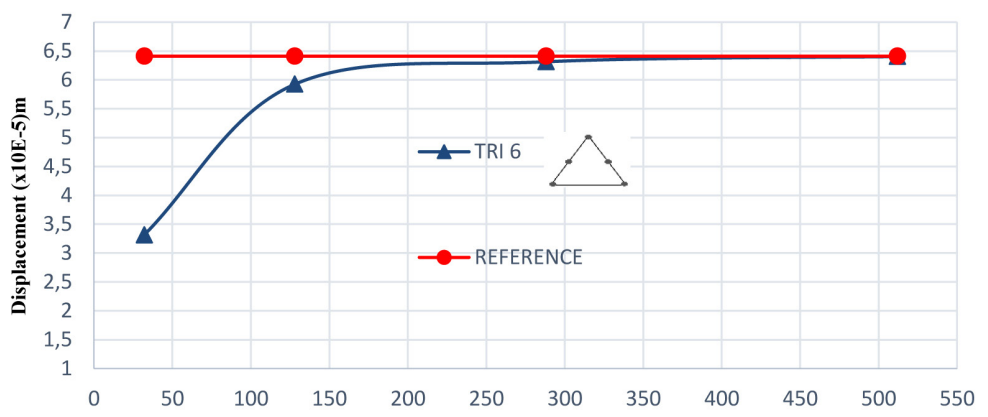

Figure 6. Graph of Displacement vs Number of elements for Triangular simply supported 


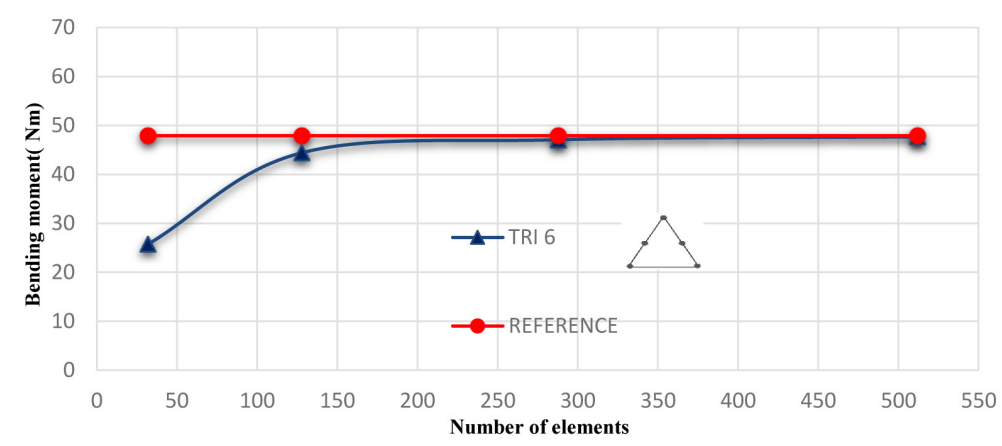

Figure 7. Graph of Bending Moment vs Number of elements for Triangular simply supported

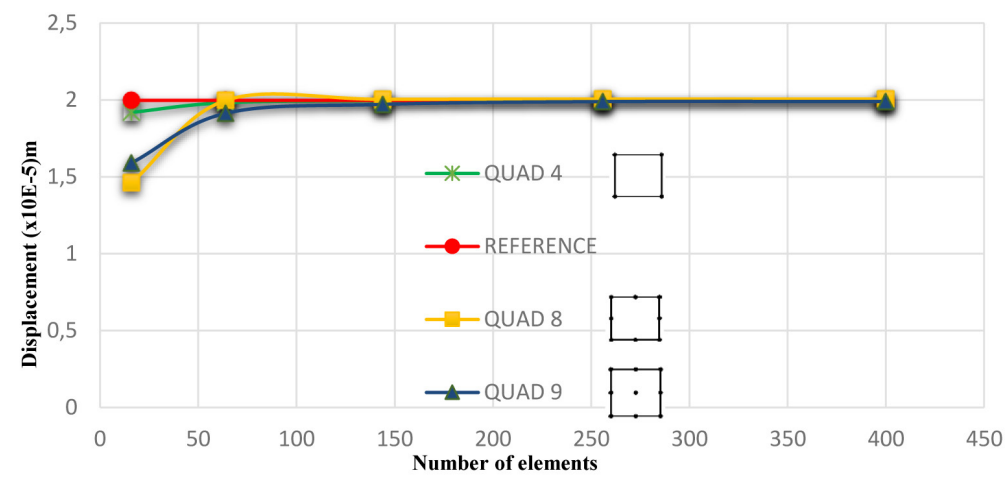

Figure 8. Graph of Displacement vs Number of elements for quadrangular, fixed supported.

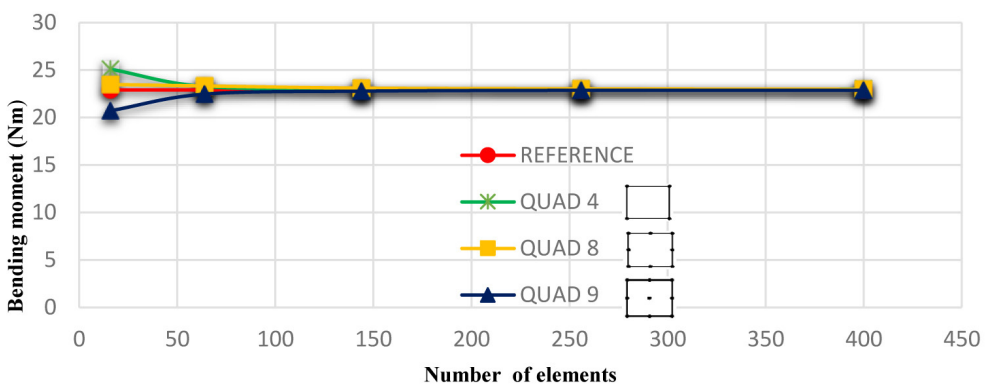

Figure 9. Graph of Bending Moment vs number of elements for quadrangular, fixed supported

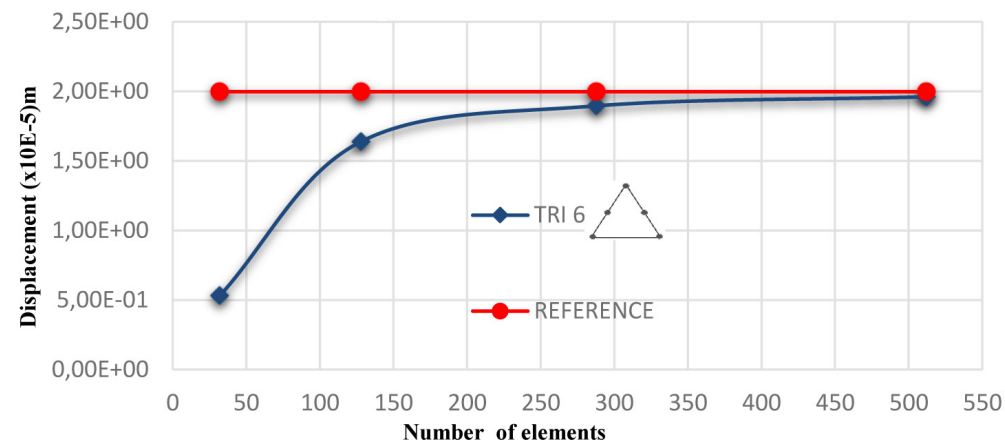

Figure 10. Graph of Displacement vs Number of elements for Triangular, fixed supported 


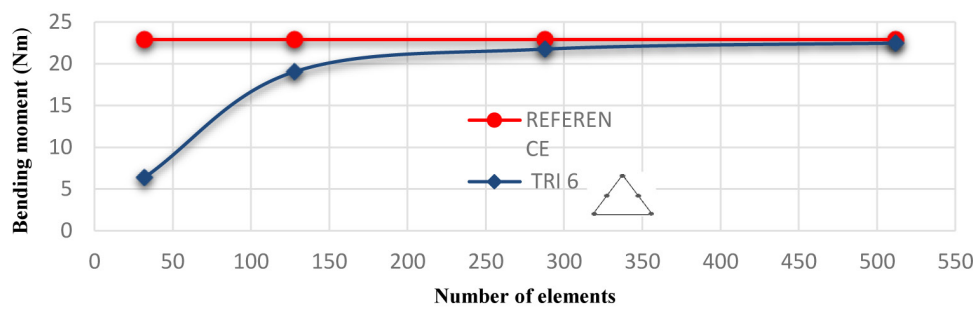

Figure 11. Graph of Bending Moment vs Number of elements for Triangular, fixed supported.

Table 3. Recommended ratios for quadrangular elements of various nodes numbers.

\begin{tabular}{|c|c|}
\hline \multicolumn{2}{|c|}{ Quadrangular (Quad) } \\
\hline & Ratio \\
\hline Quad 4 & 0.006945 \\
\hline Quad 8 & 0.015625 \\
\hline Quad 9 & 0.062500 \\
\hline
\end{tabular}

Table 4. Recommended ratios for triangular elements of various nodes numbers.

\begin{tabular}{|c|c|}
\hline \multicolumn{2}{|c|}{ Triangular (Tri) } \\
\hline & \\
\hline Tri6 & 0.003472 \\
\hline
\end{tabular}

Table 5. Recommended ratios for quadrangular elements of various nodes numbers.

\begin{tabular}{|c|c|}
\hline \multicolumn{2}{|c|}{ Quadrangular (Quad) } \\
\hline & Ratio \\
\hline Quad 4 & 0.062500 \\
\hline Quad 8 & 0.062500 \\
\hline Quad 9 & 0.015625 \\
\hline
\end{tabular}

Table 6. Recommended ratios for triangular elements of various nodes numbers.

\begin{tabular}{|c|c|}
\hline \multicolumn{2}{|c|}{ Triangular (Tri) } \\
\hline & Ratio \\
\hline Tri6 & 0.003472 \\
\hline
\end{tabular}

A quadrangular element with 9 nodes exhibited a good accuracy compared to the others in terms of displacement.

According to the results obtained, the following recommendations regarding the objectives set are made. For general use, the ratios of element mesh size to the size of the whole square plate was established for each element type and support system which met a targeted error of $5 \%$ so that to be relied on, for any dimensions of a given square plate in bending with the same aspect ratio and targets to achieve the same error value.
Consider a square plate with $\mathrm{x}^{*} \mathrm{y}$ dimensions and an element type with $\mathrm{a}$ *b dimensions that met the targeted error mentioned above, the ratio of mesh size of an element to the size of the whole plate will be ratio $=\frac{a b}{x y}$. Therefore, for a new square plate with $\mathrm{w}^{*} \mathrm{z}$ dimensions and with the same aspect ratio as the reference square plate, for it to meet an error of $5 \%$, the size of an single element will be $\frac{a b}{x y} \times w z$. The ratios established in Tables 3-6 are recommended for relevant element type and support system.

\section{REFERENCES}

1. James D., Finite Element Approach to Reinforced Concrete Slab Design. A Thesis Presented in Partial Fulfillment for the Degree of Science in Masters of Engineering, Georgia Institute of Technology., 2005.

2. Wegmuller A.W., Finite Element Analyses of Elasic-Plastic Plates and Eccentrically Stiffened Plates. The research Reported During the Author's Graduate Study for the Ph.D Degree in Civil Engineering at Lehigh University, Bethlehem,Pennsylavia, 1971.

3. Zienkiewicz O C., The Finite Element Method in Structural and Continuum Mechanics,Second Edition, New York: McGraw-Hill, 1970.

4. Carlton D., Application of the Finite Element Method to Structural Engineering Problems, The structural Engineer Vol 71, No 4 Pages 55-59, February, 1993.

5. Skorpen S.A., Comparison of Different Methods of Analysis,Dessign and Detailing of Reinforced Concrete Footing. Project Report in Partial Fulfillment for The Degreeof Masters of Engineering (structural Engineering), University of Pretoria, july,2013.

6. Reddy J.N., An Introduction to Finite Element 3rd Edition, New York : McGraw-Hill, 2005.

7. Reddy J.N., Theory and Analysis of Elastic Plates and Shells .Second Edition, CRC Press, 2007. 
8. Love A.E.H., A Treatise on the Mathematical Theory of Elasticity.Fourth edition ., Dover, 1944.

9. Timoshenko S.-K. S., Theory of Plates and Shells. Second edition, McGraw-Hill, 1959.

10. Meleshko V.V., Bending of An Elastic Rectangular Clamped Plate : Exact Versus Engineering Solutions. vol. 48, no. 1-50, 1997.

11. Lim C.W., W.A.Yao and S.Cui, Benchmark Symplectic Solutions for Bending of Corner Supported Rectangular Thin Plates: Civil and Structural Engineering. vol. 1, no. 2 january 2008.

12. Batista M., New Analytical Solution for Bending Problem of Uniformly Loaded Thin Rectangular Plate Supported on Corner Points. vol. 3, no. Civil and Structural Engineering, May,2010.

13. Vinson J.R., Structural Mechanics: The Behavior of Plates and Shells, Wiley, 1974.

14. Zienkiewicz OC; Brotton DM; Morgan L, The Finite Element Primer for Structural Engineers. Structural Engineer, Vol 54, No. 10, 1976, 387-397.

15. Brooker O., How to Design Reinforced Concrete Flat Slabs using Finite Element Analysis, Camberley: The Concrete center, 2006.
16. Argyris J., The Analysis of Complex Elasic Structures. Appl.Mech.Rev.,II, 1958.

17. Washizu K., Variational Methods in Elasticity and Plasticity. Oxford: Pergam Press, 1960.

18. British Standard, "BS 8110:1997,Structuraluse of Concrete,Part 1,Code of Practise for Design and Construction".

19. Cook R., Concepts and Application of Finite Element Analysis. New York : John Wiley \& Sons ,Inc.,fourth edition., 2002.

20. Hossel S., Comparisonof Differents Methods of Analysis. Design and Detailing of Reinforced Concrete Footing. Project Report in Partial Fullfilment for the Degree of Bachelor of Engneering, University of Pretoria, Pretoria, 2012.

21. Jones A.M., Flat Slab Design: Past, Present and Future. Structures \& Building, Vol 158,Issue SB2 pages 133-140, April,2005.

22. Hrabok M.M. and T. M. Hrudey, Structural Modelling of Stiffened Plates for Analysis and Design. Advances in Structural Engineering Computing.

23. Hatheway A.E., A review of finite element analysis techniques: capabilities and limitations. Optomechanical Design: A Critical Review, Dec. 1992. 